ppi $201502 Z U 4645$

Esta publicación científica en formato digital es continuidad de la revista impresa ISSN-Versión Impresa 0798-1406 / ISSN-Versión on line 2542-3185Depósito legal pp $197402 Z$ U34
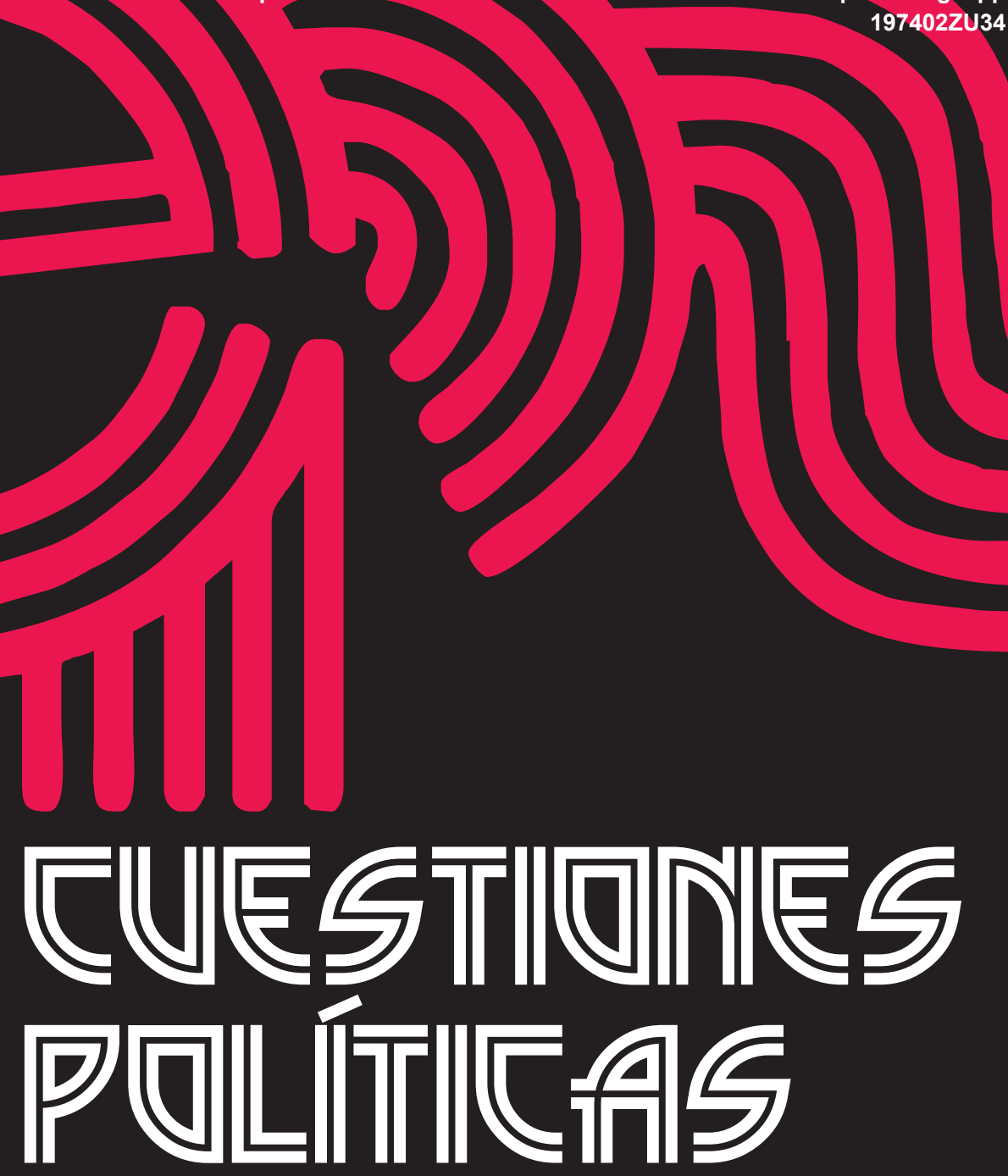

Instituto de Estudios Políticos y Derecho Público "Dr. Humberto J. La Roche" de la Facultad de Ciencias Jurídicas y Políticas de la Universidad del Zulia Maracaibo, Venezuela
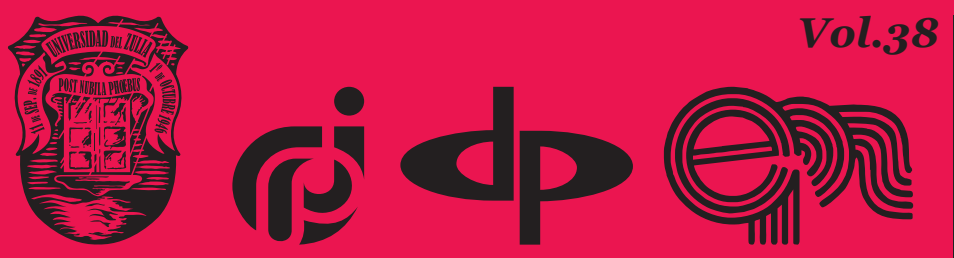

$N^{\circ}$ Especial 2da Parte 2020 


\title{
Conflictividad política, pandemia de COVID-19 y nuevos paradigmas
}

\author{
DOI: https://doi.org/10.46398/cuestpol.382e.oo
}

\author{
Jorge Jesús Villasmil Espinoza *
}

\section{Resumen}

Todo indica que el año 2020 va a terminar signado por altos niveles de conflictividad política que pudieran configurar un escenario internacional de entropía exponencial con resultados inciertos para el orden y la estabilidad global. Si a eso le sumamos los estragos económicos y sociales generados por la pandemia del nuevo coronavirus el panorama que se vislumbra para el 2021 no es nada alentador. No obstante, la pregunta obligada a responder por los científicos sociales y filósofos en general es ¿̇pueden estos acontecimientos configurar nuevos paradigmas políticos, económicos y socioculturales en el mundo? El objetivo de estas reflexiones radica en presentar el número especial, Vol. 38 , II parte, y al mismo tiempo analizar los escenarios de conflictividad política en el mundo actual en el contexto del COVID-19. A modo de conclusión se destaca el hecho de que, a pesar de las expectativas de justicia social, democracia y Derechos Humanos de buena parte de las sociedades humanas, no se vislumbran en lo discursos de las elites académicas los elementos teóricos y epistemológicos necesarios para configurar nuevos o al menos renovados modelos de organización política, económica y social más allá de los trajinados sociales y liberalismos de antaño.

Palabras clave: conflictividad política; pandemia de COVID-19; nuevos paradigmas; editorial de Cuestiones Políticas; estabilidad global. 


\title{
Political conflict, COVID-19 pandemic, and new paradigms
}

\begin{abstract}
Everything indicates that the year 2020 will end up signified by high levels of political conflict that set up an international scenario of exponential entropy with testimonies for order and global stability. Add to that the economic and social havoc generated by the pandemic of the new coronavirus, the outlook for 2021 is not encouraging at all. However, the question for social scientists and philosophers in general is these events to shape new political, economic and sociocultural paradigms in the world? The objective of reflections lies in presenting in the special issue, Vol. 38 , II part, and at the same time analyzing the scenarios of political conflict in the real world in the context of COVID-19. The conclusion highlights the fact that, in addition to the expectations of social justice, sorrow and human rights of much of human societies, the theoretical and epistemological elements necessary to shape new or at least models of political, economic and social organization beyond social and liberal trajinos are not apparent in what academic elites.
\end{abstract}

Keywords: political conflict; pandemic COVID-19; new paradigms; Editorial of Political Issues; global stability.

\section{Presentación número especial (II parte)}

Nuestra hipótesis que afirma que todo indica que el año 2020 va a terminar signado por altos niveles de conflictividad política que pudieran configurar un escenario internacional de entropía exponencial con resultados inciertos para el orden y la estabilidad global, se sustenta al menos en 3 acontecimientos puntuales por su trascendencia y significación internacional: a) el conflicto por los resultados de las elecciones presidenciales de EUA realizadas el pasado martes 03 de noviembre del año en curso; b) la guerra entre Armenia y Azerbaiyán por el control efectivo de la región Nagorno Karabaj denominada por los armenios como república de Artsaj y; c) el proceso constituyen adelantado en Chile que modificará la constitución instaurada por la dictadura militar en 1980.

En el primer caso, las elecciones presidenciales en EUA, la democracia paradigmática del mundo occidental desde sus orígenes en el siglo de las luces se evidencia que las poliarquías contemporáneas puede ser una estructura frágil incluso en sociedades como la norteamericana que han 
desarrollado históricamente un sofisticado sistema institucional para su resguardo y adaptación a los desafíos que impone cada coyuntura. Basta con que algunos actores o factores de poder propaguen, con el debido fundamento o sin él, la duda sobre la legitimidad de los resultados del ritual electoral para que se erosione la confianza en las instituciones republicanas.

En este contexto de incertidumbre, el presidente Donald Trump afirma sistemáticamente que él es el ganador de la contiende electoral y que es víctima de un fraude orquestado por el partido demócrata y su candidato ganador Joseph Robinette Biden Jr, con la anuencia de las grandes cadenadas mediáticas de ese país. ¿̇posee el presidente Trump las evidencias necesarias que avalen su reclamo o, por el contrario, se trata de los desvaríos de un personaje egocéntrico que no ha podido asimilar su derrota? En el momento que se escriben estas líneas todavía es muy prematuro para saberlo, sin embargo, queda claro que sobre la democracia norteamericana y su sistema electoral se ha posicionado una atmosfera de sospecha.

En el segundo caso, la guerra entre Armenia y Azerbaiyán por el control efectivo de la región Nagorno Karabaj, guerra que se ha venido sucediendo en varios episodios desde el ocaso de la URSS, con resultados nefastos en perdida de vidas humanas, desplazados y violación a los derechos humanos de la población civil de armenios y azeríes por igual, evidencia que los mecanismos diplomáticos tradicionales no son suficientes para reducir los resentimientos etno-políticos de países que se perfilan como enemigos históricos.

Además, estas conflagraciones bélicas entre estados son de nuevo el escenario propicio para que potencias como Rusia y Turquía interesadas en el control de la región transcaucásica interfieran en el conflicto para sacar provecho de este, aunque siendo justos, la intervención de Rusia ha sido más, hasta el momento, la de un mediador que la de un factor beligerante y su despliegue de tropas en Nagorno Karabaj es parte del acuerdo para cesar el conflicto (infobae, 2020). Como fue la tendencia general en la guerra fría podemos suponer que: ¿Estos conflictos deparan el inicio de un conjunto de guerras proxy en la región? ¿Realmente busca Turquía desarrollar un proyecto hegemónico neo-otomano? Tal como señala Taspinar (2009).

El tercer caso aludido, tiene que ver con el proceso constituyente que se esta desarrollando en este momento en Chile, otrora país modelo por su estabilidad política y económica en la América meridional. Tal como explica Morales Quiroga (2020), el estallido social experimentado por el país austral el 18 de octubre de 2019 y que echo por tierra la estabilidad del país por más de dos meses se explica por la acumulación de varias crisis:

Primero, una crisis de participación que se vio agudizada por la instauración del voto voluntario en 2012. Segundo, una crisis de representación reflejada en la 
menor adhesión a partidos y en la pérdida de confianza a instituciones claves de la de democracia (gobierno, tribunales, congreso). Tercero, una crisis de confianza en instituciones de orden público y de orden social (Carabineros e Iglesias). Cuarto, una crisis de probidad pública y privada reflejada en el incremento de casos de financiamiento irregular de las campañas políticas y colusiones empresariales (Morales, 2020: 03).

En este escenario dialéctico que conjuga a fuerzas contrarias democráticas y antidemocráticas, la sociedad civil organizada y autoconvocada en las manifestaciones de descontento junto el liderazgo político del presidente Sebastián Piñera coincidieron en adelantar un proceso constituyente como condición de posibilidad para gestionar la crisis y, fundamentalmente, construir de forma concertada otro contrato social que proporciona a las mayorías un umbral superior de justicia social y desarrollo con equidad.

Ante esta situación se justifican las siguientes preguntas: ¿Es una nueva constitución suficiente para superar los problemas de participación democrática, representación política y confianza institucional que erosionan a la democracia chilena? ¿Puede ser el proceso constituyen el caldo de cultivo para la posterior emergencia de liderazgo populistas-radicales que personalizan la política en detrimento del marco institucional? Esperamos de corazón que este no sea el caso, aunque en Latinoamérica abunden ejemplos que apuntan en sentido contrario.

Específicamente, la selección de artículos que resulto del arbitraje para publicar en este número da cuenta de excelentes investigaciones internacionales que reflejan legitimas preocupaciones jurídicas y políticas en íntima relación con el complejo mundo actual. Apertura el número en la sección Ciencia Política la investigación Civil Identity of Youth in the Conditions of Regional Social and Political Tensión de la autoría de Iana Alexeevna Siruikova y Evgeniya Valer'evna Khramova el cual se planteó por objetivo analizar el proceso de construcción de la identidad civil de la juventud en condiciones de tensión social y política, desde la perspectiva regional en Rusia.

En la sección Ciencia Política y desarrollo social el artículo de la autoría de Ruslan Rustamovich Ibragimov y otros, intitulado Religious Associations in Tatar Assr under Conditions of Mass Political Repressions of the 1930 se plateo por objetivo en clave de historia política contemporánea, analizar la situación de las asociaciones confesionales en la República Socialista Soviética Autónoma de tártaro, durante el período de represiones políticas masivas de fines de la década de 1930. En la misma sección, Anvar Ajratovich Gafarov y Mariam Arslanovna Galeeva estudian los aspectos sociopolíticos de la peregrinación musulmana rusa a La Meca en el siglo XIX en su investigación Socio-Political Aspects of Russian Muslim Pilgrimage to Mecca in the XIX-Th Century. 
Seguidamente, en la sección Ciencia política y desarrollo político, Guzel Anvarovna Valeeva y colaboradores presentan los resultados de su investigación en un artículo intitulado Security In Rights as a Variety of National Security el cual tuvo por objetivo determinar los signos de seguridad jurídica sobre la base de los puntos de vista científicos existentes y la legislación vigente, excluyendo su comprensión excesivamente amplia. En la misma sección, Sabina Rafailevna Efimova y Evgeniya Valerevna Khramova en su trabajo Socio-Political Tension in the Russian Society: Case Study of Youths of the Republic of Tatarstan exploran el nivel de tensión sociopolítica en Tartaristán. El objeto del estudio es el grupo social juvenil como portador de activismo social. Por último, Elena M. Chertakova y otros en su trabajo Special features of vocational training institutions in the context of pandemics analizan la experiencia de implementar la educación a distancia en el contexto de la pandemia de coronavirus.

En la sección Teoría política, Ghasem Shariatikia y otros presentan un sugestivo trabajo intitulado Identifying the Mental Model of the Managers of Melli Bank Regarding Quantum Leadership using Q-methodology el cual tuvo por objetivo Identificar el modelo mental de los gerentes de Melli Bank en relación con el llamado liderazgo cuántico utilizando la metodología Q.

En la sección Filosofía política Ainur Razifovich Gilmullin y Eduard Vladimirovich Krasnov exponen su investigación Unity of law and legal act as a key principle of the rule of law con el objetivo de discutir uno de los principios clave del estado de derecho, como lo es el principio de la unidad de la ley. En consecuencia, se analizaron las opiniones de estudiosos que definen este principio y destacan sus principales características conceptuales. En la misma sección, Jaafar Naser Abdulridha y otros, presentan un trabajo intitulado Legal basis for the interaction between local with state authorities con el objetivo de analizar la base legal para la interacción entre autoridades locales y estatales en el marco de lo que estipula la constitución vigente de la república de Irak.

También en la misma sección Filosofía política, Jaafar Naser Abdulridha y colaboradores discuten la filosofía de la disuasión privada en el marco del código penal en su trabajo The philosophy of private deterrence in the penal code que da cuenta de una problemática jurídica y al mismo tiempo política. Asimismo, en esta sección, Oleksandr V. Kozachenko y otros presentan una investigación intitulada Taxonomy of compulsory and incentive legal consequences (legal measures) of committing illegal acts la cual se planteó por objetivo elaborar una taxonomía de las consecuencias legales obligatorias e incentivadoras de cometer actos ilegales. 
En la sección Política internacional Ruslan Rustamovich Ibragimov y otros estudian las relaciones Estado e iglesia ortodoxa rusa en el contexto de la segunda guerra mundial y los primeros años de postguerra en un interesante trabajo intitulado Foreign policy factor in State-Church relations in the Soviet Union during World War II and early post-war. En la misma sección, Victoria Ravilevna Sagitova y Andrey Valeryevich Ivanov presentan su investigación Conflicts associated with migratory processes: a political perspective la cual tuvo por objetivo discutir el carácter conflictivo de los procesos migratorios que se dan en el mundo de hoy. Por último, en la misma sección, Marat Zufarovich Galiullin y colaboradores presentan una investigación denominada Political, Military and Technical Cooperation of the Republic of Uzbekistan in the Framework of the Shanghai Cooperation Organization con el objetivo de analizar de los principales vectores de cooperación técnico-militar de la República de Uzbekistán en el marco de la Organización de Cooperación de Shanghai (OCS) en la región de Asia Central.

Por su parte, en la sección Participación, democracia y gobernabilidad, Hryhorii Bukanov y otros exponen los resultados de una investigación denominada Training and implementation of the environmental, economic, and legal development policy of the regions: main practice-oriented approaches que se planteo por objetivo estudiar las posibilidades de los enfoques orientados a la práctica en la administración pública, en el campo de la formación e implementación de la política de desarrollo ecológico de las regiones, entre las cuales destacan, las condiciones sociales y económicas modernas de Ucrania.

Seguidamente, en la sección Informática jurídica Damir Khamitovich Valeev y Anas Gaptraufovich Nuriev en su investigación Implementation of the Function of Maximizing the Security Level in the Administration of Justice in the Digital Economy analizan la implementación de la función de maximización del nivel de seguridad en la administración de justicia en el contexto de la economía digital. En la misma sección, Timur Giorgievich Okriashvili y otros se plantearon por objeto de estudio definir los activos digitales como una categoría legal y también precisar sus características para distinguir los objetos digitales de otros objetos civiles según las características identificadas en un interesante trabajo intitulado Digital Objects: The Legal Peculiarities, the Role and Prospects of Use.

En la misma sección informática jurídica, el artículo Distance and Online Learning Solutions in the Context of Modern Legal Educational Policy de la autoría de Raviya Faritovna Stepanenko y colaboradores analiza e identifica las mejores estrategias y modelos de educación a distancia y en línea aplicables a las universidades rusas 
en el contexto de la política educativa legal moderna, considerando su efectividad. También en esta sección Kateryna Danchenko y Olga Taran presentan un artículo denominado Criminal Liability of Medical Profecionals Negligience: Comparative Analysis, el cual tuvo por objetivo identificar áreas de especializaciones donde la negligencia médica ocurre con mayor frecuencia con base a la jurisprudencia de Ucrania (los datos del único registro estatal de decisiones judiciales de Ucrania de 2016 a 2019). De igual modo, el trabajo de Olga Trishchuk y colaboradores intitulado Internet Media During the COVID-19 Crisis se planteo por objetivo definir las principales características de los medios durante la cuarentena, para analizar la peculiaridad de los medios online durante la crisis provocada por la pandemia de coronavirus COVID-19.

En la sección Reforma del estado, Ruslan Faritovich Garipov y Denis Ivanovich Igonin presentan su artículo Restriction of the Rights of Russian Senators as a Political Responsibility con el objetivo de explorar las características de la implementación de los derechos parlamentarios individuales por parte de los miembros del Consejo de la Federación y la cámara alta de la asamblea legislativa rusa.

En la sección Cultura política y participación electoral, destaca la investigación de Boris Perezhniak y colaboradores Electronic technologies during local elections: new challenges desarrollada con el objetivo de estudiar el papel de las tecnologías electrónicas durante las elecciones locales en Ucrania.

De seguida en la sección Derechos humanos, Lyaysan Renatovna Mustafina y otros presentan un trabajo intitulado Social and legal guarantees of the rights of convicts according to the legislation of the Russian Federation desarrollado con el objetivo de discutir las garantías sociales y legales de los derechos de los condenados según la legislación de la Federación de Rusia. Por otra parte, en la sección Derecho público y participación social, Stanislav Igorevich Golubev y colaboradores en su indagación denominada Environmental crime: dialectical and criminological visión examinan el estado, las causas $\mathrm{y}$ algunas medidas para prevenir los delitos ambientales.

En la sección Derecho público, Alexander Yurevich Epihin y otros presentan un trabajo denominado The investigator as an independent subject of criminal prosecution in cases of terrorist crimes con el fin de estudiar la teoría y la práctica del enjuiciamiento penal de delitos terroristas en la Federación de Rusia. En la misma sección, Ildar A Tarhanov y colaboradores identifican las cuestiones fundamentales de libertad condicional en el derecho penal de la Federación de Rusia y, al mismo tiempo, discuten los aspectos del impacto negativo del encarcelamiento en la identidad del reo, desde varios puntos de vista sobre el programa de "ascensores sociales". 
En esta misma sección, Gulshan Gurezovna Bodurova y Sanavbar Nazirkulovna Tagaeva describen las tendencias de desarrollo dela regulación legal vinculada a colisiones que regulan los asuntos matrimoniales bajo las leyes de los países postsoviéticos en un interesante artículo titulado Tendencies for the Development of Marriage Conflict Legal Regulation with the Participation of Foreigners and Persons without Citizenship in the Post-Soviet Space. Del mismo modo, el artículo Features of Qualification and Prevention of Smuggling of Narcotic Drugs, Psychotropic Substances: their Precursors or Analogues de la autoría de Khayrullina Rezeda Gazinurovna y otros, analiza la legislación rusa en función de ofrecer opciones para resolver problemas en el campo del contrabando de estupefacientes, sustancias psicotrópicas, sus precursores y análogos.

Igualmente, en la misma sección, Jaafar Naser Abdulridhay Ghani Ressan Gadder presentan un estudio intitulado The problems of organization and legal responsibility (civil and administrative) in the field of telecommunications in Iraq con la finalidad de describir los problemas de organización y responsabilidad legal (civil y administrativa) en el campo de las telecomunicaciones en Irak. Aquí mismo, Hedayatollah Soltani Nezhad y Javad Hazeri exponen los resultados de la investigación Constituent elements of prohibited vertical agreements in the competition law of Iran and the European Union desarrollada con la finalidad de analizar los elementos constitutivos de los acuerdos verticales prohibidos por la ley de competencia de Irán y, con lo que se establece en la ley de competencia de la Unión Europea.

Por su parte, Alexander Sergeevich Baleevskikh y Oleg Ilyasovich Katlishin en su investigación State regulation efficiency of food companies of Russia in sanction period describen el nuevo curso político de la Federación de Rusia, destinado a superar las sanciones económicas de los países de Europa, así como de los Estados Unidos al país euroasiático. En esta misma sección derecho público, Motliakh Oleksandr y colaboradores en su trabajo Mediation in criminal proceedings: legal regulation in Ukraine and foreign experience of application estudian las peculiaridades del uso de la institución de mediación en los procesos penales de los países europeos para implementar esta experiencia positiva en la legislación de Ucrania. Del mismo modo, el artículo de investigación Dynamics of the implementation of the protective role in the conduct of crimes: the practice of the Convention for the Protection of Human Rights and Fundamental Freedoms de la autoría de Dariia Balobanova y colaboradores analiza la dinámica de la implementación de la función protectora en la condena por delitos en la interpretación de la Corte Europea de Derechos Humanos. 
En esta misma sección, el articulo intitulado Jurisprudence of the European court of human rights in the choice of precautionary measures in criminal proceedings: legal realities and perspectives de la autoría de Svitlana Romantsova y otros, discute los problemas de aplicación de las decisiones del TEDH en la selección de medidas cautelares en casos penales de conformidad con la legislación nacional. Dado que la legislación procesal de Ucrania actualmente no es perfecta en el marco del establecimiento y regulación de la aplicación de medidas cautelares, las decisiones del TEDH sirven como un regulador indispensable de esta cuestión. Además, destaca el trabajo de investigación Victimological measures to prevent violent offences for gain committed by children de la autoría de Volodymyr Shablystyi y colaboradores que tuvo por objetivo proporcionar una descripción victimológica de los delitos violentos con fines lucrativos cometidos por niños en Ucrania.

Por su parte, la investigación de Marcia Fabiola Jaramillo Paredes y otros, intitulada Influencia de la innovación en la competitividad de las medianas empresas del Ecuador tuvo por finalidad analizar la influencia de la innovación en la competitividad de las medianas empresas del Ecuador. Asimismo, y en la misma sección, el artículo de Nana Bakaianova y colaboradores Information technology in the litigation due to the pandemic COVID-19 examinan las tecnologías de la información en litigio y su desarrollo en la pandemia del nuevo coronavirus.

En la misma sección, Tetiana Blashchuk y colaboradores presentan un artículo intitulado Virtual Mediation in the Field of Intellectual Property con la finalidad de analizar los aspectos teóricos del uso de las tecnologías de la información en el proceso de mediación, así como estudiar los puntos problemáticos de su implementación. También, Ernest Gramatskyy y otros, en su investigación Innovation IT-Payment Technologies as a Know-how and an Object of Intellectual Property Rights realizan un análisis de la introducción y funcionamiento del know-how, para determinar los vectores de su uso, teniendo en cuenta las necesidades de los participantes en las relaciones jurídicas que surgen en este ámbito.

Por último, Denysiuk Stanislav y colaboradores en su trabajo Foreign experience in professional development of private detectives analizan la experiencia internacional en el desarrollo profesional de detectives privados, con el fin de implementar algunos aspectos positivos en la legislación de Ucrania. Igualmente, Kozin Serhii y otros cierran el presente número especial, con su artículo Description of the legal basis for the protection of labor rights of migrants el cual tuvo por objetivo analizar el marco legal para la protección de los derechos laborales de los migrantes, identificando un rango específico de normativas relacionadas con esta categoría y considerando sus principales disposiciones en Ucrania. 


\section{Referencias Bibliográficas}

INFOBAE. 2020. "Rusia desplegó tropas en Nagorno Karabaj como parte del acuerdo para cesar el conflicto entre Armenia y Azerbaiyán". Disponible en línea. En: https://www.infobae.com/america/mundo/2020/11/11/ rusia-desplego-tropas-en-nagorno-karabaj-como-parte-del-acuerdopara-cesar-el-conflicto-entre-armenia-y-azerbaiyan/. Fecha de consulta: 17/11/2020.

TASPINAR, Ömer. 2009. "Entre neootomanismo y kemalismo La política exterior turca en Oriente Próximo se debate entre el neootomanismo, partidario de una ambiciosa visión geoestratégica, y el kemalismo, que opta por la moderación" En: Gran Angular. Disponible en línea. En: https://www.iemed.org/observatori/arees-danalisi/arxius-adjunts/ afkar/afkar-idees-21/9Taspinar.pdf/. Fecha de consulta: 17/11/2020.

QUIROGA MORALES, Mauricio. 2020. "Estallido social en Chile 2019: Participación, representación, confianza institucional y escándalos públicos" En: Análisis Político. No. 98, enero-abril, pp. 3-25. 

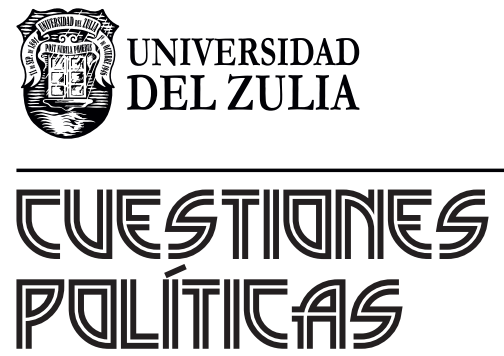

Vol.38 NEspecial

Esta revista fue editada en formato digital y publicada en diciembre de 2020, por el Fondo Editorial Serbiluz, Universidad del Zulia. Maracaibo-Venezuela 